\title{
Size of Supramolecular SNARE complex: Membrane-Directed Self-Assembly
}

\author{
Won Jin Cho, Aleksandar Jeremic, and Bhanu P. Jena* \\ Wayne State University School of Medicine, Department of Physiology, Detroit, MI, USA 48201
}

\begin{abstract}
All life processes are governed at the chemical level. Knowledge of how single molecules interact, provides a fundamental understanding of Nature. An aspect of molecular interactions, is the self-assembly of supramolecular structures. For example, membrane fusion requires the assembly of a supramolecular complex formed when proteins in opposing bilayers interact. Membrane fusion is essential for numerous cellular processes, including hormone secretion, enzyme release, or neurotransmission. In living cells, membrane fusion is mediated via a specialized set of proteins present in opposing bilayers. ${ }^{1,2}$ Target membrane proteins, SNAP-25 and syntaxin (t-SNAREs) and secretory vesicle-associated protein (v-SNARE), are part of the conserved protein complex involved in fusion of opposing lipid membranes. ${ }^{1,2}$ The structure and arrangement of membraneassociated full length SNARE complex, was first examined using atomic force microscopy (AFM). ${ }^{3}$ Results from the study demonstrate that t-SNAREs and v-SNARE, when present in opposing bilayers, interact in a circular array to form supramolecular ring complexes each measuring a few nanometers. ${ }^{3}$ The ring-complex helps in establishing continuity between the opposing bilayers. ${ }^{3}$ In contrast in the absence of membrane, soluble $\mathrm{v}$ - and t-SNAREs fail to assemble in any specific pattern, or form such conducting pore structures. ${ }^{3}$
\end{abstract}

SNARE-ring complexes ranging in size from approximately $15 \mathrm{~nm}$ to $300 \mathrm{~nm}$ in diameter are formed when t-SNARE-reconstituted and v-SNARE-reconstituted lipid vesicles meet. Since vesicle curvature would dictate the contact area between opposing vesicles, this broad spectrum of SNARE complexes observed, may be due to the interaction between SNAREreconstituted vesicles of different size. To test this hypothesis, t-SNARE- and v-SNAREreconstituted liposomes (proteoliposomes) of distinct diameters were used. Lipid vesicles of different sizes used in the study were isolated using published extrusion method. ${ }^{4,5}$ The size of each vesicle population was further assessed using the AFM (Fig. 1). AFM section analysis demonstrates the presence of small 40-50 nm-in diameter vesicles isolated using a $50 \mathrm{~nm}$ extruder filter (Fig. 1A, B). Similarly, representative samples of large vesicles measuring 150-200 nm and 800-1000 nm were obtained using different size filters in the extruder. Such large vesicles are shown in the AFM micrograph (Fig. 1C, D). Analysis of vesicle size using photon correlation spectroscopy, further confirmed the uniformity in the size of vesicles within each vesicle population (data not shown).

*bjena@med.wayne.edu.

Supporting Information Available: Experimental procedures is available via the internet at http://pubs.acs.org 
The morphology and size of the SNARE complex formed by the interaction of t-SNAREand v-SNARE-reconstituted vesicles of different diameter were examined using the AFM (Fig. 2). In each case, the t-SNARE and v-SNARE proteins in opposing proteoliposomes, interact and self-assemble in a circular pattern, forming pore-like structures. The interaction and arrangement of SNAREs in a characteristic ring pattern were observed for all populations of proteoliposomes examined (Fig. 2A-D). However, the size of the SNARE complex was found to be dictated by the diameter of the proteoliposomes used (Fig. 2). When small $(\sim 50 \mathrm{~nm})$ t-SNARE- and v-SNARE-reconstituted vesicles were allowed to interact, SNARE-ring complexes of $\sim 20 \mathrm{~nm}$ in diameter were generated (Fig. $2 \mathrm{~A}, \mathrm{~B}$ ). With increase in the diameter of proteoliposomes, larger t-/v-SNARE complexes were formed (Fig. 2 C, D). A strong linear relationship between size of the SNARE complex and vesicle diameter is demonstrated from these studies (Fig. 3). The experimental data fit well with the high correlation coefficient, $\mathrm{R}^{2}=0.9725$ between vesicle diameter and SNARE-complex size (Fig. 3).

Unlike calculated values, which fundamentally assume vesicles to be non-deformable and hard spheres, our experimental data suggest that these artificial lipid vesicles, similar to secretory vesicles, are soft. Hence, once v-SNARE and t-SNAREs from opposing vesicles meet, the initial SNARE complex formed, pulls the opposing bilayers closer to each other. As a consequence, vesicles become flattened, which then leads to an increase in contact area between the opposing vesicles. The result is a further increase in t-/v-SNARE contacts, allowing the formation of larger SNARE-ring complexes. In case of hard vesicles, flattening would be unlikely, and therefore result in forming smaller SNARE-ring complexes. On the other hand, deformation of soft vesicles leads to an increase in contact area between the opposing bilayers and a resultant increase in Gibbs free energy. In an elastic membrane, the surface free energy is given by the equation: $(1 / 2) \mathrm{k}_{\mathrm{a}}(\Delta \mathrm{A})^{2} / \mathrm{A}_{0}$, where $\mathrm{k}_{\mathrm{a}}$ is the bending modulus, $\Delta \mathrm{A}$, the increase in surface area, and $\mathrm{A}_{0}$, the initial unstressed area. ${ }^{6}$ Therefore, an increase in surface area results in an increase in the Gibbs free energy, and the spontaneous fusion between opposing bilayers become less probable. ${ }^{6-8}$ Hence, large vesicles are less fusogenic than smaller vesicles. This would explain why neurons being fast secretory cells, possess small 40-50 nm in diameter vesicles, for rapid and efficient fusion and release of neurotransmitters at the nerve terminal, ${ }^{9,10}$ compared to a slow secretory cell like the exocrine pancreas, with larger (200-1,200 nm in diameter) secretory vesicles, for the slow and prolonged release of digestive enzymes. ${ }^{11,12}$

In summary, we have demonstrated that the size of a self-assembled supramolecular SNARE protein complex can be controlled. This has implications in the regulated fusion of artificial lipid membranes, which may find use in the controlled delivery of lipid-encapsulated drugs and the transport of molecules.

\section{Supplementary Material}

Refer to Web version on PubMed Central for supplementary material. 


\section{Acknowledgment}

Supported by NIH grants (BPJ).

\section{References}

1. Söllner T, Whiteheart SW, Brunner M, Erdjument-Bromage H, Geromanos S, Tempst P, Rothman JE. Nature. 1993; 362:318-324. [PubMed: 8455717]

2. Weber T, Zemelman BV, McNew JA, Westerman B, Gmachi M, Parlati F, Söllner TH, Rothman JE. Cell. 1998; 92:759-772. [PubMed: 9529252]

3. Cho SJ, Kelly M, Rognlien KT, Cho J, Hoerber JKH, Jena BP. Biophys. J. 2002; 83:2522-2527. [PubMed: 12414686]

4. MacDonald RC, MacDonald RI, Menco BP, Takeshita K, Subbarao NK, Hu LR. Biochim. Biophys. Acta. 1991; 1061:297-303. [PubMed: 1998698]

5. Jeremic A, Kelly M, Cho JA, Cho SJ, Horber JK, Jena BP. Cell Biol. Int. 2004; 28:19-31. [PubMed: 14759765]

6. Israelachvili, J. Intermolecular and surface forces. 2nd Ed.. Academic Press; San Diego, CA: 1992.

7. Ohki SJ. Memb. Biol. 1984; 77:265-275.

8. Wilschut J, Duzgunes N, Papahadjopoulos D. Biochemistry. 1981; 20:3126-3133. [PubMed: 7248275]

9. Cho WJ, Jeremic A, Rognlien KT, Zhvania MG, Lazrishvili I, Tamar B, Jena BP. Cell Biol. Int. 2004; 28:699-708. [PubMed: 15516328]

10. Kelly M, Cho WJ, Jeremic A, Abu-Hamdah R, Jena BP. Cell Biol. Int. 2004; 28:709-716. [PubMed: 15516329]

11. Jena BP, Schneider SW, Geibel JP, Webster P, Oberleithner H, Sritharan KC. Proc. Natl. Acad. Sci. U.S.A. 1997; 94:13317-13322. [PubMed: 9371843]

12. Schneider SW, Sritharan KC, Geibel JP, Oberleithner H, Jena BP. Proc. Natl. Acad. Sci. U.S.A. 1997; 94:316-321. [PubMed: 8990206]

$J$ Am Chem Soc. Author manuscript; available in PMC 2015 September 23. 

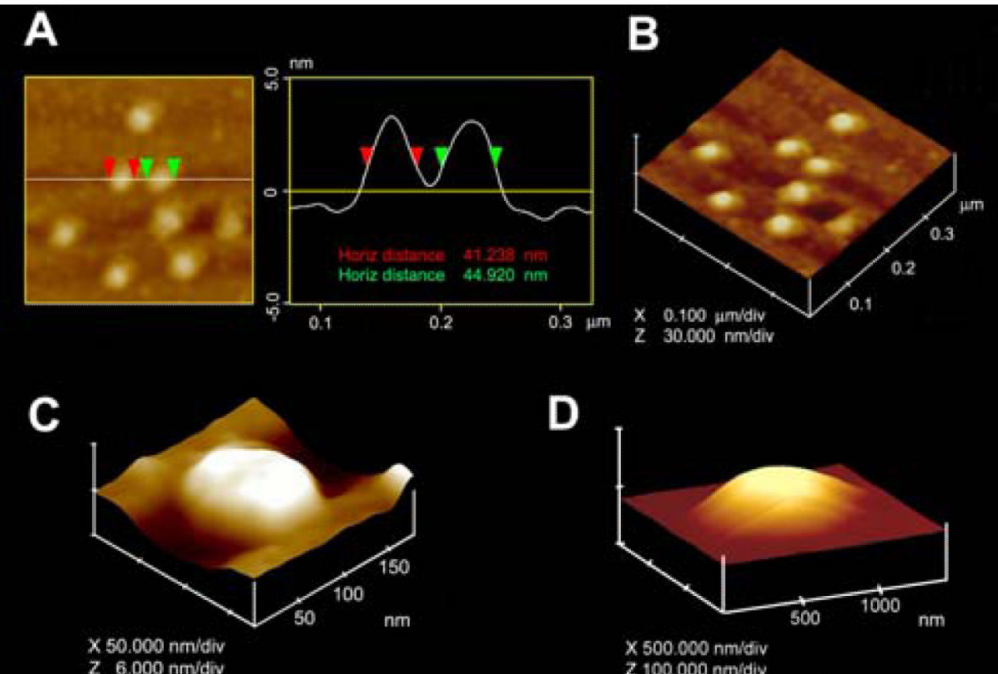

D

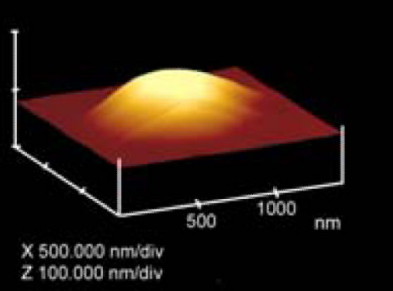

Figure 1.

AFM micrograph of t-SNARE and v-SNARE reconstituted liposomes of different sizes. Note the $\sim 40-50 \mathrm{~nm}$ vesicles (A,B), the $\sim 150 \mathrm{~nm}$ (C) and $\sim 800 \mathrm{~nm}$ vesicle (D). 


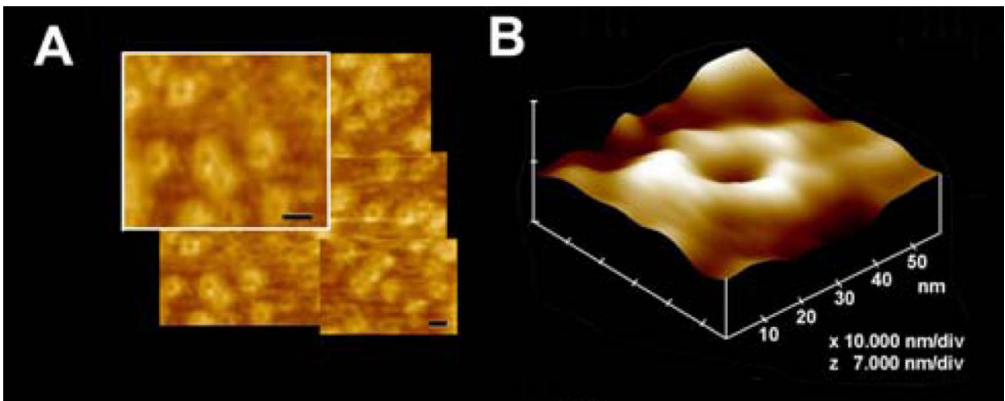

C

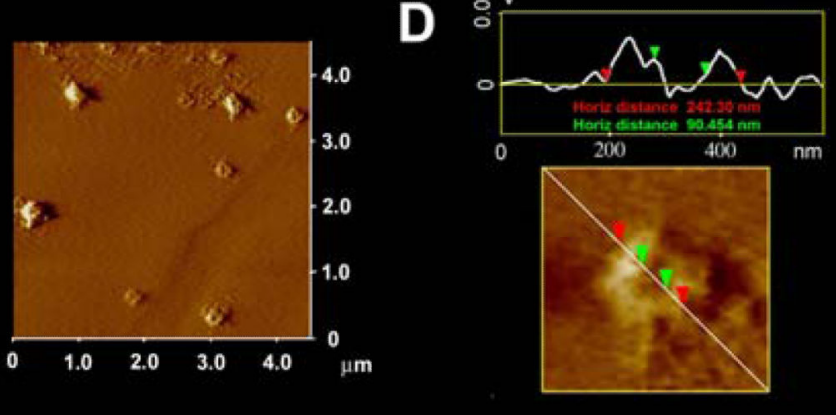

Figure 2.

Representative AFM micrograph of t-/v-SNARE complexes formed when small (A, B) or large (C, D) t-SNARE and v-SNARE reconstituted vesicles interact with each other. Note the formation of different size SNARE complexes, which are arranged in a ring pattern. Bar= 20nm. AFM section analysis (D) shows the size of a large SNARE complex. 

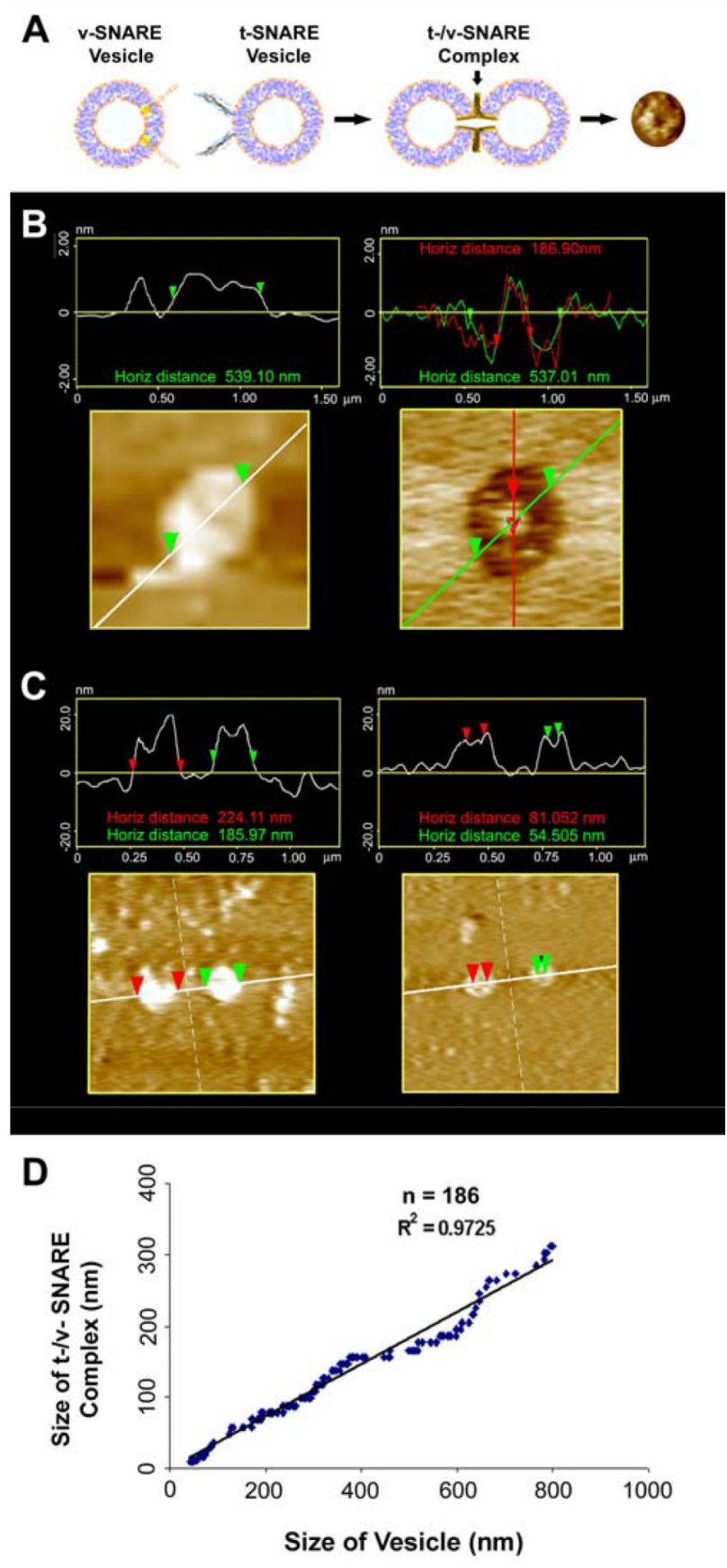

Figure 3.

SNARE complex is directly proportional to vesicle diameter. Schematic diagram depicting the interaction of $t-S N A R E$ and $v-S N A R E$ reconstituted vesicles. At the extreme right, is a single $t-/ v-S N A R E$ complex imaged by AFM (A). AFM images of vesicles before and after their removal by the AFM cantilever tip, exposing the t-/v-SNARE complex (B). Interacting t-SNARE and v-SNARE vesicles imaged by AFM at low $(<200 \mathrm{pN})$ and high forces (300-500 pN). Note, at low imaging forces, only the vesicle profile is imaged (left $\mathrm{C}$ ). However at higher forces, the soft vesicle is flattened, allowing the SNARE complex to be 
imaged (right C). Plot of vesicle diameter vs. size of the SNARE complex. Note the high correlation coefficient $\left(\mathrm{R}^{2}=0.9725\right)$ between vesicle diameter and the size of the SNARE complex (D). 